\title{
Comparison of KOH Mount Using Standard Technique and Cellophane Tape Method for Diagnosis of Superficial Fungal Infections
}

\author{
B. Navya* and Saroj Golia \\ Department of Microbiology, Dr. B.R. Ambedkar Medical College, KG Halli, \\ Bengaluru, Karnataka, India \\ *Corresponding author
}

\begin{tabular}{|l|}
\hline Ke y w or d s \\
Standard technique, \\
Cellophane tape \\
method, KOH \\
mount, Malassezia \\
species, \\
Dermatophytes.
\end{tabular}

A B S T R A C T

Superficial fungal infections include Tinea versicolor and Dermatophytosis. Tinea versicolor infects stratum corneum which is caused by lipophilic yeast-like fungi i.e. Malassezia species. Dermatophytosis infects keratinised structures (skin, hair and nails) caused by a group of keratinophilic fungi called dermatophytes. $\mathrm{KOH}$ mount is the preliminary, convenient and inexpensive test for diagnosis of superficial fungal infections. This study compares the detection rate and visualisation of fungal elements by $\mathrm{KOH}$ mount using standard technique and cellophane tape method. This study was carried out in the Department of Microbiology, Dr B R Ambedkar Medical College, K G Halli, Bangalore for a period of 6 months from August 2016 to January 2017. A total of 250 skin scrapings were collected from clinically diagnosed patients with superficial fungal infections (Tinea versicolor- 76, Dermatophytosis- 174). Preliminary $\mathrm{KOH}$ mount examination was done by standard technique. The same patients were also studied by a modified technique by using cellophane tape method for $\mathrm{KOH}$ mount. Out of 250 clinical samples, 71 were positive for Malassezia species, 170 were positive for Dermatophytes and 9 were negative for fungal elements. Standard technique and cellophane tape method of $\mathrm{KOH}$ mount both were positive for fungal elements in $91.6 \%$ of samples. Standard technique of $\mathrm{KOH}$ mount was negative but cellophane tape method was positive in $4.8 \%$ of samples. Both the methods were negative in $3.6 \%$ of the samples. The present study concludes that there is a higher rate of detection and the morphology of fungal elements were better visualised by cellophane tape method of $\mathrm{KOH}$. Also, the standard technique of $\mathrm{KOH}$ mount is time consuming compared to cellophane tape method. Cellophane tape method of $\mathrm{KOH}$ mount is easier and convenient for collection and transport of specimen as well. Thus, cellophane tape method of $\mathrm{KOH}$ mount serves as a better preliminary diagnostic tool than the standard conventional technique.

\section{Introduction}

The most common cutaneous fungal infections in the world are Tinea versicolor and Dermatophytosis. It is more common in tropical countries (63\%) like India due to factors like heat and humidity, over population and poor hygienic conditions.
The high humidity and temperature provides a fertile ground for the abundant growth of these fungi ${ }^{2,9}$. Patients with a propensity for developing Tinea infections may have a genetic component that predisposes them to disease, either immunosuppression or 
hyperhidrosis ${ }^{1,4}$. Tinea versicolor is caused by Malassezia species which is a lipophilic yeast, which are part of the normal skin flora. They sometimes assume a pathogenic state within the epidermis.

When this occurs, patients will present with hypopigmented and/or hyperpigmented scaling lesions on the upper trunk independent of the immune status of the patients ${ }^{1,4}$.The other contributing factor is colonization of Malassezia species in anatomically vulnerable areas of the skin. Exogenous factors such as sunlight, corticosteroids, and oil-based products encourage the growth of Malassezia yeast forms.

Dermatophytes form a closely related group of keratinophilic fungi, causing infection of the superficial keratinized horny layer of skin, the hair and the nails and do not cause systemic infection ${ }^{3}$.Dermatophytosis are widespread both in developed and developing countries particularly due to the advent of immunosuppressive drugs, frequent usage of antibiotics and various conditions like organ transplantations, lymphomas, leukaemia and Human immunodeficiency virus (HIV) infection ${ }^{5}$.

The clinical presentation, though very typical of fungal infection, is very often confused with other skin disorders particularly due to rampant application of broad-spectrum steroid containing skin ointments and creams leading to further misdiagnosis and mismanagement. So, there is a need for correct, rapid and efficient laboratory methods for diagnosis of these fungal infections ${ }^{9}$.

$\mathrm{KOH}$ mount is the preliminary, convenient and inexpensive screening test done for the diagnosis of superficial fungal infections. It can be done as an outpatient procedure and the results are available within minutes. Only drawback is that it requires an expert to identify and appreciate fungal elements and report the same. It is the most useful and reliable test with the result of which the Dermatologist can start an empirical treatment based on the morphological differences of fungal elements seen in Tinea versicolor and Dermatophytosis. Further confirmation can be done by culture and biochemical reactions which help in speciation of the isolates.

The main of this study includes, finding the age and sexing distribution of superficial fungal infections in the study population. And to compare the methods of $\mathrm{KOH}$ mount using standard technique and cellophane tape method.

The objectives of this study includes, to compare the detection rate and visualisation of fungal elements from samples of superficial fungal infection patients by $\mathrm{KOH}$ mount using standard technique and cellophane tape method and to compare the time taken for reporting of the same by these methods.

\section{Inclusion criteria}

Clinically diagnosed cases of Tinea versicolor and Dermatophytosis.

Cases of all age groups and both sexes.

\section{Exclusion criteria}

Clinical diagnosis of Tinea nigra.

\section{Materials and Methods}

A study was undertaken with 250 clinical samples collected from clinically diagnosed patients of superficial fungal infections (Tinea versicolor-76, Dermatophytosis-174) attending the Dermatology and Venereology outpatient department of Dr B R Ambedkar Medical College and Hospital, Bangalore during a period of 6 months from August 
2016 to January 2017. The diagnosis of superficial fungal infection was based on the clinical history and physical examination. This study was carried out in the Department of Microbiology, Dr B R Ambedkar Medical College and Hospital, Bangalore.

\section{Sample collection}

The affected area was cleaned aseptically with $70 \%$ ethyl alcohol and allowed to dry. Skin sample was collected directly on to a sterile black paper envelope using a preflamed blunt scalpel from the edge of the lesion for standard technique 6 . The same patients were also studied by a modified method by applying a transparent cellophane tape over similar lesion, pressed firmly and removed. The tape was stuck on a sterile glass slide over which 3-4 drops of $10 \% \mathrm{KOH}$ solution was placed.

\section{Microscopic examination}

Preliminary examination was done by direct microscopy by $\mathrm{KOH}$ mount. It was done by adding 2-3 drops of $10 \% \mathrm{KOH}$ solution to the collected sample on a sterile glass slide and a cover slip was placed. The slide was observed for fungal elements after allowing 15-20 minutes for digestion and clearing of keratin making the fungal elements evident.

The same patients were also studied by a modified method using cellophane tape for collection of sample. This slide was observed immediately on receiving in the laboratory considering the fact the time required for digestion of keratin is covered in the time taken for transport of the slide.

For samples of which $\mathrm{KOH}$ mount were positive by cellophane tape method but negative by standard technique were confirmed further by culturing the same sample on to antibiotic incorporated Sabouraud dextrose agar (SDA) media with chloramphenicol $50 \mathrm{mg} / \mathrm{L}$ and cycloheximide $500 \mathrm{mg} / \mathrm{L}$ for dermatophytes and with a sterile olive oil overlay on to SDA with antibiotics for Malassezia species.

\section{Results and Discussion}

$\mathrm{KOH}$ was positive with short hyphae and round yeast cells ("spaghetti and meatball" appearance) in case of Tinea versicolor and with long septate hyphae in case of Dermatophytes. Out of 250 clinical samples, 71 were positive for Malassezia species and 170were positive for Dermatophytes and 9 were negative for fungal elements.

The majority of patients were of the age group 20-40 years constituting $60.4 \%$ of which males were $36.4 \%$ and females were $24 \%$.

Followed by the age group $<20$ years constituting $30 \%$ of patients and $9.6 \%$ of patients were of age group $>40$ years. On the whole, males (62.4\%) were more affected than females $(37.6 \%)$ by superficial fungal infections in this study (Table 2).

Standard technique and cellophane tape method of $\mathrm{KOH}$ mount for detecting fungal elements both were positive for $91.6 \%$ of samples. Standard technique of $\mathrm{KOH}$ mount was negative but cellophane tape method positive for $4.8 \%$ of samples. Both the methods were negative for $3.6 \%$ of the samples (Table 1).

Also, the morphology of fungal elements was better visualised by cellophane tape method compared to standard technique. All of the 12 samples which were $\mathrm{KOH}$ mount negative by standard technique but positive by cellophane tape method showed positive culture growth on SDA with antibiotics thus confirming the validity of the cellophane tape method. In the present study with 250 clinical samples, 229 $(91.6 \%)$ were positive for fungal elements by 
both the methods under study whereas 12 $(4.8 \%)$ of the samples were positive for fungal elements only by cellophane tape method but negative by standard technique and $9(3.6 \%)$ of samples were negative for fungal elements by both methods (Figs 1-5).
Similarly, a study conducted by Thirumurthy $\mathrm{M}$ et al., ${ }^{10}$ showed that out of 15 clinical samples tested by both methods all were positive for fungal elements by both methods but fungal morphology was better appreciated by cellophane tape method.

Table.1 Comparison of standard technique of $\mathrm{KOH}$ mount with cellophane tape method

\begin{tabular}{|c|c|c|}
\hline Standard technique of KOH Mount & Cellophane tape method of KOH Mount & Results Total no. (\%) \\
\hline Positive & Positive & $229(91.6 \%)$ \\
\hline Negative & Positive & $12(4.8 \%)$ \\
\hline Negative & Negative & $9(3.6 \%)$ \\
\hline
\end{tabular}

Table.2 Age and gender distribution of superficial fungal infections

\begin{tabular}{|c|c|c|c|c|c|}
\hline \multirow{2}{*}{ Age (years) } & \multicolumn{2}{|c|}{ Tinea versicolor } & \multicolumn{2}{c|}{ Dermatophytosis } & \multirow{2}{*}{ Total } \\
\cline { 2 - 5 } & Male & Female & Male & Female & \\
\hline \hline$<20$ & 13 & 8 & 32 & 22 & $75(30 \%)$ \\
\hline $20-40$ & 21 & 23 & 70 & 37 & $151(60.4 \%)$ \\
\hline$>40$ & 8 & 3 & 12 & 1 & $24(9.6 \%)$ \\
\hline Total & 42 & 34 & 114 & 60 & $250(100 \%)$ \\
\cline { 2 - 5 } & \multicolumn{2}{|c|}{$76(30.4 \%)$} & $174(69.6 \%)$ & \\
\hline
\end{tabular}

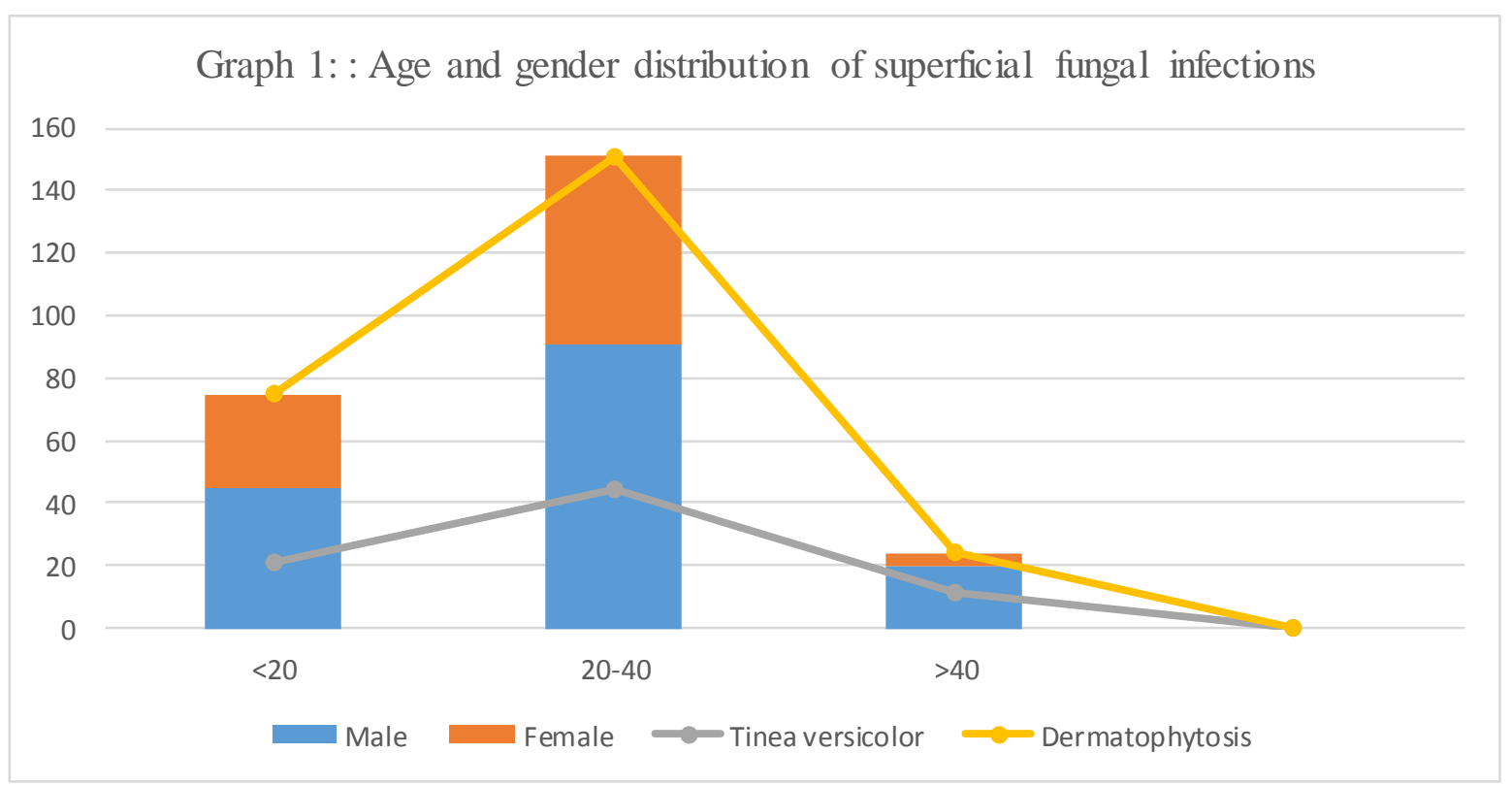


Fig.1 Slides of $\mathrm{KOH}$ mount by standard technique and cellophane tape method

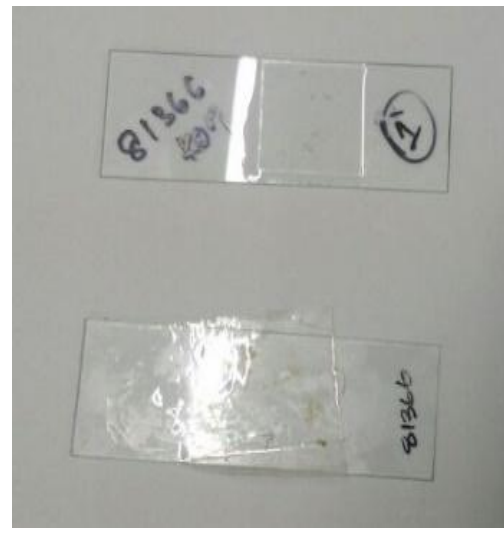

Fig.4 KOH mount of Dermatophytosis by standard technique
Fig.2 KOH mount of Tinea versicolor by standard technique
Fig.3 KOH mount of Tinea versicolor by cellophane tape method

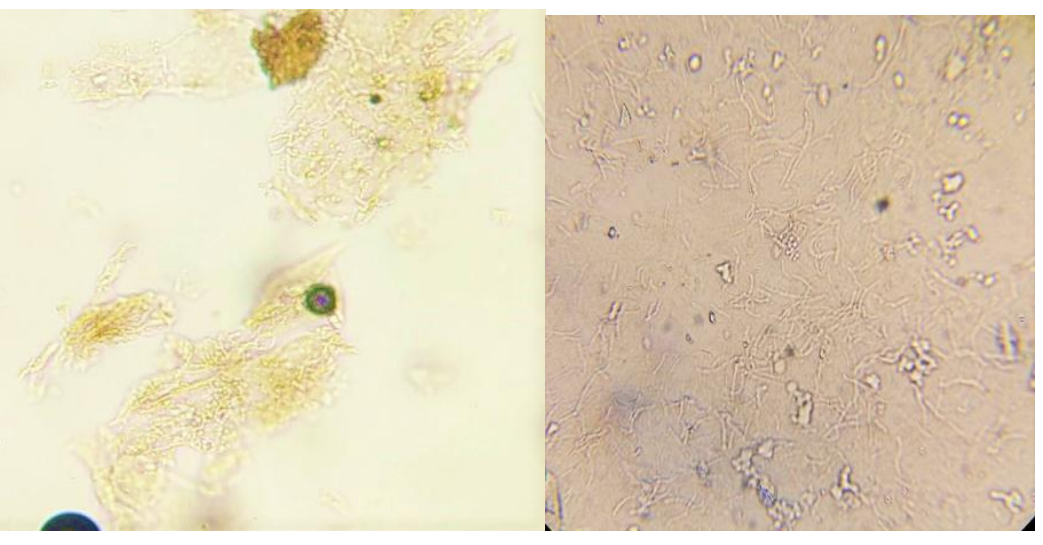

Fig.5 KOH mount of Dermatophytosis by cellophane tape method

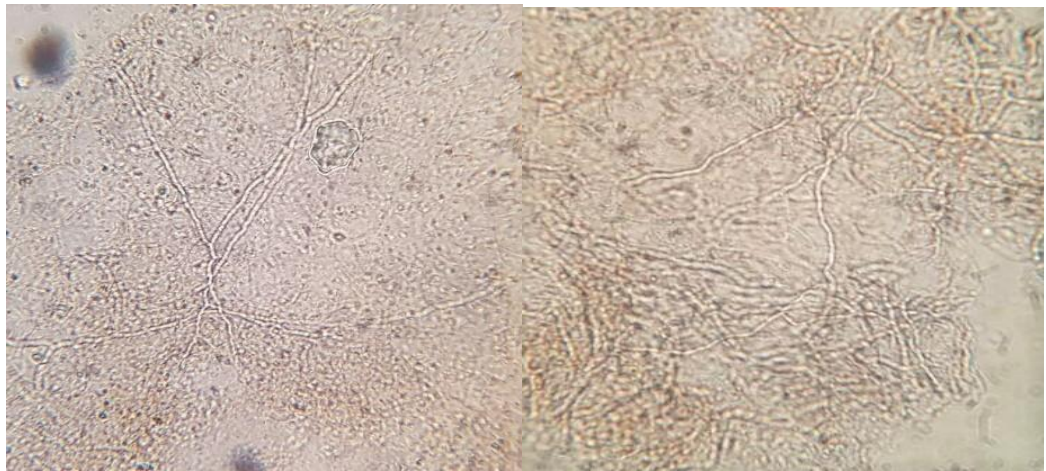

As per the study by Kurade et al., ${ }^{7}$ transportation of sample by cellophane tape method is easier compared to the standard technique. Milne et al., ${ }^{8}$ studied the use of cellophane tape method for detection of deep seated cutaneous mycoses after the usefulness of this method for Malassezia species, Candida species and dermatophytes was proved.

The present study concludes that there is a higher rate of detection by cellophane tape method compared to standard technique of $\mathrm{KOH}$ mount. Also, the morphology of fungal elements was better visualised by cellophane tape method of $\mathrm{KOH}$ mount reason being the hyphae remain undisturbed in the sample. The standard technique of $\mathrm{KOH}$ mount is time consuming compared to cellophane tape method as it takes 15-20 minutes for $\mathrm{KOH}$ solution to digest the keratin material and reveal the fungal elements. Whereas by the cellophane tape method fungal elements are detected within few minutes of slide reaching the laboratory as $\mathrm{KOH}$ is added at the time of collection of specimen itself.

Cellophane tape method of $\mathrm{KOH}$ mount is easier and convenient for collection and transport of specimen as well. Cellophane tape method also has an advantage of collecting good amount of sample especially 
when only scanty amount of sample is available for collection by scraping the edge of the lesion for standard technique of $\mathrm{KOH}$ mount. Thus, cellophane tape method of $\mathrm{KOH}$ mount serves as a better preliminary diagnostic tool than the standard conventional technique.

\section{Acknowledgement}

It is with great privilege and respect that I express my most cordial and humble thanks to my respected teacher and guide, Dr. (Mrs). Saroj Golia MD, Professor and HOD Department of Microbiology, Dr. B.R. Ambedkar Medical College Bangalore. I owe my indebtedness for her unparalleled and excellent guidance, continuous encouragement, in working on this study. I feel fortunate to work under her. I express sincere appreciation to my senior colleagues Dr Jyoti and Dr Suhani S Manasa for their support and co-operation. I express deep sense of gratitude to all the patients who cooperated for the study.

\section{References}

Allen HB, Goyal K, Ogrich L, Joshi S (2015). Biofilm Formation by Malassezia Furfur/Ovale as a Possible Mechanism of Pathogenesis in Tinea versicolor. J Clin Exp Dermatol Res. 6:311.

Anjana G, Divya H, Arun K R (2015). Mycological profile of dermatophytes isolated from clinical samples in KIMS Hospital, Bangalore. JEMDS. 4(5);835842.
Dey NC, Grueber HLE, Dey TK (2006). Cutaneous mycosis, Dermatophytosis. In: Chapter 29, Medical Mycology. $1^{\text {st }}$ edition, Central Publications, Kolkata, India. 295-356.

Gupta AK, Lane D, Paquet M (2014). Systematic Review of Systemic Treatments for Tinea versicolor and Evidence-Based Dosing Regimen Recommendations. J Cutan Med Surg. 18: 79-90.

Kannan P, Janaki C, Selvi GS (2006). Prevalence of dermatophytes and other fungal agents isolated from clinical samples. Indian J Med Microbiol. 24(3): $212-5$

Kumar BCS, Gopi A, Harindranath D, Gupta D, Hitha TK, UlKhair SM (2015). Isolation and Speciation of Malassezia in Patients Clinically Suspected of Pityriasis Versicolor. Int J Sci Stud. 3(6):18-23.

Kurade SM, Amladi SA, Miskeen AK (2006). Skin scraping and a potassium hydroxide mount. Indian $\mathbf{J}$ Dermatol Venereol Leprol. 72:238-41.

Milne LJR, Barnetson R St. C (1974). Diagnosis of dermatophytoses using vinyl adhesive tape. Sabouraudia. 12:162-165.

Ragavendra Rao M, Rashmi Mahale P, Tejashree A, Rajeshwari K G, Madhuri Kulkarni (2015). Evaluation of culture media for rapid isolation of dermatophytes in JSS Medical College, Mysore: IOSR-JDMS. 14(8):06-09.

Thirumurthy M, Sethuraman G, Srinivas C R (2002). KOH mount for superficial fungal infections using cellophane tape: Comparison with standard technique. Indian J Dermatol Venereol Leprol. 68:136.

\section{How to cite this article:}

Navya, B. and Saroj Golia. 2017. Comparison of KOH Mount Using Standard Technique and Cellophane Tape Method for Diagnosis of Superficial Fungal Infections. Int.J.Curr.Microbiol.App.Sci. 6(10): 494-499. doi: https://doi.org/10.20546/ijcmas.2017.610.060 